\title{
Lucky numbers: Spatial neglect affects physical, but not representational, choices in a Lotto task
}

\author{
Tobias Loetscher ${ }^{1,2}$ \\ Michael E.R. Nicholls ${ }^{1}$ \\ John N. Towse ${ }^{3}$ \\ John L. Bradshaw ${ }^{4}$ \\ Peter Brugger ${ }^{2}$
}

1 School of Behavioural Sciences, University of Melbourne, Melbourne, Australia

2 Department of Neurology, University Hospital Zurich, Zurich, Switzerland

3 Psychology Department, Lancaster University, Lancaster, U.K.

4 School of Psychology, Psychiatry and Psychological Medicine, Monash University, Australia

MANUSCRIPT INFORMATION:

Total number of pages: 18

Number of words: 3419 (main text)

Number of Figures: 1

To appear in the journal Cortex. Please consult this journal for a final, authoritative version.

Corresponding author: Tobias Loetscher, School of Behavioural Sciences, University of Melbourne, Parkville, VIC 3010, Australia; phone ++61 38344 4303; FAX: ++61 39347 6618; e-mail: tobias.loetscher@alumni.ethz.ch 


\begin{abstract}
Spatial neglect can be characterized by a "magnetic attraction" towards the right side of a visual stimulus array and a selection of stimuli from that hemispace. This study examined whether these distinctive characteristics in visuo-motor space are also evident in representational number space. Given that numbers are thought to be represented along a leftto-right oriented mental number line, an affinity for the spontaneous selection of larger numbers was anticipated for neglect patients. Contrary to this expectation, neglect patients $(n=20)$ picked a similar range of numbers compared to controls $(n=17)$ when generating a number between 1,000 and 10,000 and when playing an imaginary lottery game. There was, however, a positive correlation between the biases for the imaginary lottery, number generation and a number bisection task - demonstrating that exploration asymmetries along the mental number line are consistent within individuals across tasks. Some of the patients selected smaller numbers in all of these tasks, confirming reports of dissociations between physical and numerical-representational forms of neglect. Conversely, only four $(20 \%)$ of the patients could reliably be classified as demonstrating a neglect in number space. When filling out a physical lottery ticket, the neglect patients showed the expected bias towards picking numbers placed on the right-hand side of the ticket. These results demonstrate that the magnetic attraction towards the right side of mental representations is rather weak and that representational forms of neglect only occasionally co-exist with neglect in physical space.
\end{abstract}

\title{
Keywords:
}

Space representation, representational neglect, spatial attention, mental number line, numerical cognition

\section{Acknowledgments}

This study was supported by a grant from the Swiss National Science Foundation to T.L. 


\section{Introduction}

The ability to shift spatial attention is fundamental for effective interactions with the world. The importance of spatial attention is particularly evident in patients suffering from right brain damage and symptoms of spatial neglect. A key characteristic in many of these patients is the automatic orienting of attention towards visual stimuli located on the ipsilesional side (D'Erme, Robertson et al., 1992; Gainotti, D'Erme et al., 1991) and the consecutive difficulties in disengaging and reorienting attention from these stimuli (Posner, Walker et al., 1984). Spatial neglect can therefore lead to bizarre behaviors such as bumping into objects placed on the left, ignoring people situated on the left or eating only the right side of a dish. Intriguingly, the disorder of spatial neglect is not restricted to physical space, but also affects the generation and/or exploration of mental images (Bisiach and Luzzatti, 1978). Thus, when neglect patients describe familiar places, maps or other geographical facts from memory, they may fail to name left-sided, but not right-sided landmarks of the imagined spatial layout (e.g., Bartolomeo, D'Erme et al., 1994; Meador, Loring et al., 1987; Rode, Rossetti et al., 2004).

In a putatively independent line of research, cognitive psychologists are concerned with how numbers are mentally represented and processed. Results of psychophysical experiments (e.g., Dehaene, Bossini et al., 1993; Moyer and Landauer, 1967; Restle, 1970) suggest that numerical magnitudes are represented on an analogue, oriented "mental number line", with small numbers placed on the left, and larger numbers on the right side of space. Zorzi, Priftis et al., (2002) tested this account of a mental number line in patients with left spatial neglect. They reasoned that, if the numbers are spatially represented on a mental number line, then neglect patients who bisect physical lines to the right of the true center, should show a similar rightward bias for mental number lines. In support of this proposition, when asked to identify the middle between two numbers (e.g. $1 \& 9$ ), neglect patients systematically placed the 
bisection to the right of true centre (e.g. 6 or 7). These findings are an impressive demonstration of the close interaction between spatial and numerical processing and are in line with research showing that space and numbers are intricately linked in the brain's organization - with the parietal lobes playing a crucial role in this association (see de Hevia, Vallar et al., 2008; Hubbard, Piazza et al., 2005; Wood and Fischer, 2008; Sandrini and Rusconi, In Press). Indeed, it has been suggested that the parietal circuits involved in shifting attention in the external world are homologous to those that shift the spotlight of attention along mental representations such as the number line (e.g., Dehaene, Piazza et al., 2003; Fischer, Castel et al., 2003; Hubbard, Piazza et al., 2005).

The current study further examined the relation between spatial disorder and number processing in neglect patients using an ensemble of novel tasks. To date, many studies that have explored attention along the mental number line have used bisection tasks, which potentially are analogous to physical line bisection tasks (see Umiltà, Priftis et al., 2009). Besides a rightward bias in line bisection tasks, neglect patients also show a "magnetic attraction" towards right-sided visual stimuli (De Renzi, Gentilini et al., 1989) and select items from that side from a range of available response alternatives (Campbell and Oxbury, 1976; Costa, Vaughan et al., 1969). For an array of horizontally arranged stimuli, neglect patients begin by naming objects on the right, whereas controls name objects on the left (Gainotti, D'Erme et al., 1991). Starting position is thought to be a particularly sensitive measure of visuo-spatial neglect (Azouvi, Samuel et al., 2002) and may detect residual impairment after apparent recovery from neglect (Campbell and Oxbury, 1976; Mattingley, Bradshaw et al., 1994). One specific aim of this study was to assess whether the distinctive features of an initial rightward orientation and selection bias in visuo-motor space can also be found in number space using a variety of tasks that allowed patients to select their response 
from a range of correct response alternatives. The tasks were designed to involve every-day numerical decision behavior without placing a high load on working memory (in contrast to the abstract randomization procedure used by Loetscher and Brugger, 2009). They were expected to provide further insight into systematic biases in numerical choice pertaining to the presence of hemispatial neglect.

The first task required participants to generate spontaneously a number between 1,000 and 10,000 (Kubovy, 1977). This apparently simple task is potentially informative because a response is highly underspecified (i.e. there is a large range to sample from) and thus offers opportunities for schemata and representations to influence response selection (Scott, Barnard et al., 2001). If numbers on the right side of the mental number line are more salient than those on the left side, a rightward bias should be observed. Second, a 'Lotto' task was administered, similar to an actual game highly popular in Switzerland. The Lotto task was administered in two forms: one provided a physical record sheet, the other required use of a mental representation of the number range on this sheet. While a rightward bias was predicted for both versions, dissociations were considered possible in some patients; such dissociations between physical and representational forms of neglect were previously described (Doricchi, Guariglia et al., 2005). Finally, a more traditional mental number line bisection task was administered. The relationship between the biases across the different tasks was assessed using a series of correlations. If asymmetries in the mental exploration of numbers are consistent within individuals, a correlation should be observed between the tasks. Given that physical and mental forms of neglect are dissociated in some individuals (Doricchi, Guariglia et al., 2005), the correlation between the tasks that tap these functions may show little or no correlation. 


\section{Methods:}

\subsection{Participants:}

Twenty patients $(17$ men) with left spatial neglect after a right hemisphere lesion and 17 control patients (12 men) without spatial neglect after a right hemisphere lesion participated in this study. The presence of spatial neglect was assessed by a series of paper and pencil tests, which included a line bisection task, a cancellation task, figure copying, reading and a figural fluency task (see Loetscher and Brugger, 2009, for task details and cut-off criteria). Inclusion criteria for the neglect group were symptoms of left-sided neglect in at least two of these tests, whereas the control group generally showed no neglect behavior in any task. The neglect group (mean age $=65$ years, S.D. $=11)$ was older than the control group (mean age $=54$ years, S.D. $=11 ; \mathrm{t}(35)=3.0, \mathrm{p}<.01)$. Importantly, however, the two groups did not differ in respect to lesion etiology, days since incident, presence of visual field defects, years of education and handedness ( $p>.2$ in all cases). Testing was performed in accordance with the ethical standards laid down in the 1964 Declaration of Helsinki. All participants provided written informed consent prior to participation.

\subsection{Numerical Tasks}

Three numerical tasks were administered to the patients. There was a time gap of at least 5 minutes between any two numerical tasks to avoid possible carry-over effects. During this time the patients performed either paper and pencil neglect tasks or other neuropsychological tests.

\subsubsection{Number Generation Task:}

In the number generation task participants spontaneously named the first number between 1,000 and 10,000 that came to mind. The question was repeated using exactly the same 
wording in case participants asked for clarification or if they produced a number outside the given range. The oral response was recorded by the experimenter.

\subsubsection{Lottery Task:}

For this experiment we adapted the Swiss Lotto game, which is very popular in Switzerland. In the original game, participants pick six numbers from a set of 45 (i.e. from numbers ranging from 1 to 45) by marking them on a lottery ticket. Six winning numbers are drawn by a random number generator and the lucky participants, whose chosen numbers are drawn, receive a monetary jackpot prize.

In our adapted lottery game there were two conditions. In the imaginary condition, participants spontaneously named six numbers from a set of 45 , which might be drawn the following weekend. Importantly, before the participants were asked to generate the numbers, it was stressed that they should try to choose numbers randomly and not to pick significant numbers like birthdays, anniversaries, street or house numbers as they might would do when playing real lotto (see Heinze and Riedwyl, 1998). Furthermore, familiarity with the game was assessed for each participant (e.g., whether the rules of the game are known by heart, how regularly they play). Oral responses were recorded by the experimenter. For each participant an imaginary lottery index was calculated as $\left[\frac{(\text { Number of responses }>23)-(\text { Number of responses }<23)}{(\text { Number of responses }>23)+(\text { Number of responses }<23)}\right] * 100$. The index scores could range from -100 to +100 . A negative imaginary lottery score indicates a bias towards the selection of smaller numbers, whereas a positive score denotes a bias towards larger numbers. Thus, a score of +100 indicates that all selected numbers were larger than the middle number (23). 
In the physical lottery condition, which took place roughly 40 minutes after the imaginary task, a real lottery ticket was presented to the participants. On the original Swiss Lotto ticket, the numbers are arranged on a 8 (rows) by 6 (columns) grid, starting with "1" on the top-left corner and the following numbers ordered by their ordinal rank in reading direction, from left to right and from top to bottom. Since there are only 45 numbers, the bottom-right corner of the grid is empty in the original ticket. We therefore changed the position of some numbers on the bottom row so that they were arranged symmetrically around the midline of our experimental ticket. This ticket was placed alongside the patient's midsagittal plane and they were asked to choose six numbers, which might be drawn next weekend. Again, participants were encouraged to pick numbers randomly and not to choose significant numbers as they might do in a real lottery game. They were told that they did not need to remember any of the previously orally generated numbers since this task would have no direct reference to the imaginary Lotto task. Like the imaginary lottery index, a physical lottery index was calculated for each participant $\left[\frac{(\text { Number of right responses })-(\text { Number of lef tresponses })}{(\text { Number of right responses })+(\text { Number of lef tresponses })}\right] * 100$. A negative physical lottery index indicates a bias to the leftward columns; a positive score denotes a rightward bias on the graphic lottery ticket. Thus, a score of +100 indicates that all marked numbers laid on the right side (columns 4 to 6 ) of the lottery ticket.

\subsubsection{Number line bisection task}

A number line bisection task was administered to a subgroup of the participants (same task as in Loetscher, Bockisch et al., 2008, inclusion of this task was decided rather late in the study). Nine of the neglect patients performed the number bisection task, in which eleven ascending 
(e.g. 1-7) and eleven descending (e.g. 7-1) one-digit number pairs were read to the patients, and they then had to guess the mid-number of the given interval. The selection of one-digit stimuli was based on the findings in Zamarian, Egger et al., (2007). For each participant, the mean deviation of the true midpoint was calculated, with negative values indicating leftward bisections towards smaller numbers and positive values indicating rightward bisections towards larger numbers.

\section{Results}

\subsection{Number Generation Task}

One participant was excluded from analysis, because he was unable to respond with a number in the correct range. The distribution of responses was remarkably similar for the neglect and control group, showing a preference for numbers in the lowest, middle, and uppermost quintiles of the numerical intervals (see top panel Figure 1). The magnitude of the generated number did not differ significantly between the neglect $(\overline{\mathrm{x}}=5278, \mathrm{SD}=3205)$ and control $\operatorname{group}(\overline{\mathrm{x}}=6328, \mathrm{SD}=3280, \mathrm{U}=131, \mathrm{p}>.32)$

\subsection{Lotto Task}

Familiarity and knowledge of Lotto rules did not differ between the neglect and control group ( $p>.25$ in both cases). Examination of the mean deviation for the imaginary Lotto task revealed a similar leftward bias for both groups (neglect: $\bar{x}=-16.3, S D=52.1$; control: $\bar{x}=$ 22.7, $\mathrm{SD}=29.4 ; \mathrm{U}=159.5, \mathrm{p}>.75)$. Four out of the 20 neglect patients picked more large than small numbers, whereas none of the 17 control patients did so (see mid panel Figure 1 for the distribution of the indices). Bayesian inferential statistics (see, Crawford and Garthwaite, (2007) confirmed that the larger-number bias in these four patients was significantly different to the controls (Bayesian $\mathrm{p}<.042$, one tailed). There was a positive 
correlation between the magnitude of the generated number between 1,000 and 10,000 and the imaginary Lotto index in the neglect- (Spearman's rho $=.49 ; \mathrm{p}<.04)$, but not the controlgroup (Spearman's rho $=-.06 ; \mathrm{p}>.8$ ). Thus, neglect patients who picked larger numbers in the imaginary Lotto task, also generated higher 4-digit numbers.

In the physical lottery task, neglect patients marked significantly more numbers placed on the right-hand side of the lottery ticked than did the control patients (neglect: $\overline{\mathrm{x}}=66.7, \mathrm{SD}=46.4$; control: $\overline{\mathrm{x}}=19.6, \mathrm{SD}=33.5 ; \mathrm{U}=96, \mathrm{p}<.03$, see bottom panel Figure 1 for distribution of indices). The magnitude of this right-side bias for the physical Lotto task was not related to the bias for the imaginary Lotto task for the neglect- (Spearman's rho $=.19 ; \mathrm{p}>.42$ ) or the control-patients $($ Spearman's rho $=.03 ; \mathrm{p}>.91)$.

\subsection{Number line bisection task}

As a group, the nine neglect patients did not show a rightward bias when bisecting numerical intervals (range from -.27 to $.44, \overline{\mathrm{x}}=.08, \mathrm{SD}=.22, \mathrm{z}=1.1, \mathrm{p}>.26$ ). There were, however, strong correlations between the deviations in the number line bisections tasks and the magnitude of the generated 4-digit number (Spearman's rho $=.77 ; p<.02$ ) and the imaginary Lotto task (Spearman's rho $=.69 ; \mathrm{p}<.04)$.

\section{Discussion}

One of the most distinctive features of spatial neglect in visuo-motor space is an initial orienting bias towards the right side of a stimulus array and then a selection bias for stimuli from that side (Azouvi, Samuel et al., 2002). The present study examined whether this bias is also evident in representational number space. In a left-to-right oriented representation of numbers, selection biases would be reflected in an affinity for the spontaneous selection of 
larger numbers from a given numerical interval. Compared to 17 control patients, however, the group of 20 patients with neglect neither picked larger numbers when choosing a number between 1,000 and 10,000 nor when playing an imaginary lottery game. In these tasks, therefore, there was no indication of a general rightward bias in number space when spontaneously selecting from an array of small and large number response alternatives. Conversely, when filling out a physical lottery ticket, the patients showed a bias towards picking numbers placed on the right-hand side of the ticket.

From these results, one might conclude that one of the most sensitive clinical measures of visual neglect - an initial rightward orienting bias - does not extend to the mental representation of numbers. Thus, while the very first selection from an array of horizontally arranged visual objects is typically from the right side (Azouvi, Samuel et al., 2002), this initial attraction towards right-sided (i.e. larger) numbers is absent. The very first spontaneously selected number from an array of response alternatives is as often a small (leftsided) as it as a large (right-sided) number (see top panel Figure 1). This finding is in line with research reported by Bartolomeo, D'Erme et al., (1994), who also failed to find an initial rightward orientation in representational space when patients described geographical maps from memory. Together these studies suggest that initial explorations with the mind's eye are not magnetically attracted towards the ipsilesional side of mental representations.

Spatial neglect is a heterogeneous disorder with a multitude of manifestations (see Adair and Barrett, 2008). Consequently, group comparisons may well fail to reveal the whole story in the pattern of data. Indeed, for the neglect patients, there was a positive correlation between the biases for the imaginary Lotto, number generation and number bisection tasks. Thus, individuals who selected numbers to the left for the imaginary Lotto task, selected leftward 
numbers in the number generation task and also bisected numerical intervals to the left (and vice versa for individuals who selected to the right). These correlations demonstrate that asymmetries in the exploration along the mental number line are consistent within individuals across the different tasks. Individuals with a rightward bias for all three tasks appear to show the signs of representational neglect, with an inattention to the left side of number space (Zorzi, Priftis et al., 2002). In contrast, individuals with a leftward bias for all three tasks may be performing like the controls, with a propensity for selecting smaller numbers. These patients thus showed a dissociation between physical and representational neglect, supporting a previous conjecture that these two forms of neglect can be dissociated (Doricchi, Guariglia et al., 2005).

In the imaginary lottery task, 4 out of the 20 neglect patients picked more large than small numbers, whereas none of the 17 controls did so. Bayesian inferential statistics (Crawford and Garthwaite, 2007) confirmed that these patients can be considered to suffer from neglect in number space. The incidence of $20 \%$ (4 out of 20) of patients suffering from neglect in physical and representational space lies in the spectrum reported by other investigators using non-numerical tasks, which range from 6\% (Bourlon, Pradat-Diehl et al., 2008) to around 30\% (Bartolomeo, Bachoud-Levi et al., 2005; Bartolomeo, D'Erme et al., 1994). While our data on the incidence of neglect in number space is in line with those of previous studies on the incidence of neglect for imagined space, it stands in contrast to the numerous articles reporting reliable rightward biases in mental number line bisection tasks (see Umiltà, Priftis et al., 2009). The many single-case and small-group studies, however, may not serve as a reliable basis for estimating the incidence of neglect in number space in stroke populations. Other studies do not report individual bisection data and do thus not allow inferences about incidence rates (e.g., Zorzi, Priftis et al., 2006). As of now, only two studies applied the 
mental bisection task to more than 10 patients suffering from neglect. In these studies a reliable rightward deviation was found in $27 \%$ (3 out of 11 patients, Doricchi, Guariglia et al., 2005 ) and 45\% (10 out of 22 patients, see table S5 in Doricchi, Merola et al., in press). Thus, in these relatively large patient samples, neglect in number space was found in fewer than half of the patients.

Even for visual-spatial neglect, the reported rate of occurrence after stroke varies from $12 \%$ to $100 \%$ (see Bowen, McKenna et al., 1999). With particular reference to the present study, a number of factors may affect estimated incidence rates of neglect in number space. In particular, tasks requiring more explicit orienting along the mental number line may more often document the presence of neglect. Furthermore, an increased salience of numbers used more frequently in everyday language (see Dehaene and Mehler, 1992) might have affected the choices in the number generation tasks. Despite this, the results of our multi-task experiment suggest that neglect in number space may be less prevalent than currently assumed. In fact, along with the studies by Doricchi and collaborators (Doricchi, Guariglia et al., 2005; Doricchi, Merola et al., in press) the present study questions the idea that a rightward bias in number space is found in a majority of patients suffering form visual-spatial neglect. Future prospective studies assessing larger, consecutive samples of patients are needed to shed more light on this issue.

To conclude, the results of the current study confirm previous research showing that, while visual exploration of and motor interactions with the real world are severely impaired in neglect, spatial exploration with the mind's eye may not be affected to a comparable extent (e.g., Bartolomeo, Bachoud-Levi et al., 2005; Bartolomeo, D'Erme et al., 1994; Bourlon, Pradat-Diehl et al., 2008; Halsband, Gruhn et al., 1985). This imbalance in the frequencies of 
visuo-motor and representational neglect supports the notion that neglect is predominantly a disorder of exogenous, stimulus-driven allocation of attention with relatively preserved endogenous, strategy-driven orienting capacities (Bartolomeo and Chokron, 2002; Natale, Posteraro et al., 2005; Sieroff, Decaix et al., 2007; Bourlon, Pradat-Diehl et al., 2008). 


\section{References:}

ADAIR JC AND BARRETT AM. Spatial neglect: Clinical and neuroscience review - a wealth of information on the poverty of spatial attention. Annals of the New York Academy of Sciences, 1142: 21-43, 2008.

AZOUVI P, SAMUEL C, LOUIS-DREYFUS A, BERNATI T, BARTOLOMEO P, BEIS JM, CHOKRON S, LECLERCQ M, MARCHAL F, MARTIN Y, DE MONTETY G, OLIVIER S, PERENNOU D, PRADAT-DIEHL P, PRAIRIAL C, RODE G, SIEROFF E, WIART L, and ROUSSEAUX M. Sensitivity of clinical and behavioural tests of spatial neglect after right hemisphere stroke. Journal of Neurology, Neurosurgery \& Psychiatry, 73: 160-166, 2002.

BARTOLOMEO P, BACHOUD-LEVI AC, AZOUVI P, and CHOKRON S. Time to imagine space: A chronometric exploration of representational neglect. Neuropsychologia, 43: 1249-57, 2005.

BARTOLOMEO P and CHOKRON S. Orienting of attention in left unilateral neglect. Neuroscience \& Biobehavioral Reviews, 26: 217-34, 2002.

BARTOLOMEO P, D'ERME P, and GAINOTTI G. The relationship between visuospatial and representational neglect. Neurology, 44: 1710-4, 1994.

BISIACH E and LUZZATTI C. Unilateral neglect of representational space. Cortex, 14: 129-133, 1978.

BOURLON C, PRADAT-DIEHL P, DURET C, AZOUVI P, and BARTOLOMEO P. Seeing and imagining the "Same" Objects in unilateral neglect. Neuropsychologia, 46: 2602-2606, 2008.

BOWEN A, MCKENNA K, and TALLIS RC. Reasons for variability in the reported rate of occurrence of unilateral spatial neglect after stroke. Stroke, 30: 1196-1202, 1999.

CAMPBELL DC and OXBURY JM. Recovery from unilateral visuo spatial neglect? Cortex, 12: 303-312, 1976.

COSTA LD, VAUGHAN G, HORWITZ M, and RITTER W. Patterns of behavioral deficit associated with visual spatial neglect. Cortex, 5: 242-263, 1969.

CRAWFORD JR and GARTHWAITE PH. Comparison of a single case to a control or normative sample in neuropsychology: Development of a bayesian approach. Cognitive Neuropsychology, 24: 343 - 372, 2007.

D'ERME P, ROBERTSON I, BARTOLOMEO P, DANIELE A, and GAINOTTI G. Early rightwards orienting of attention on simple reaction time performance in patients with left-sided neglect. Neuropsychologia, 30: 989-1000, 1992.

DE HEVIA MD, VALLAR G, and GIRELLI L. Visualizing numbers in the mind's eye: The role of visuo-spatial processes in numerical abilities. Neuroscience \& Biobehavioral Reviews, 32: 1361-1372, 2008.

DE RENZI E, GENTILINI M, FAGLIONI P, and BARBIERI C. Attentional shift towards the rightmost stimuli in patients with left visual neglect. Cortex, 25: 231-7, 1989.

DEHAENE S, BOSSINI S, and GIRAUX P. The mental representation of parity and number magnitude. Journal of Experimental Psychology: General, 122: 371-396, 1993.

DEHAENE S and MEHLER J. Cross-linguistic regularities in the frequency of number words. Cognition, 43: 129, 1992.

DEHAENE S, PIAZZA M, PINEL P, and COHEN L. Three parietal circuits for number processing. Cognitive Neuropsychology, 20: 487-506, 2003. 
DORICCHI F, GUARIGLIA P, GASPARINI M, and TOMAIUOLO F. Dissociation between physical and mental number line bisection in right hemisphere brain damage. Nature Neuroscience, 8: 1663-1665, 2005.

DORICCHI F, MEROLA S, AIELLO M, GUARIGLIA P, BRUSCHINI M, GEVERS W, GASPARINI M, and TOMAIUOLO F. Spatial orienting biases in the decimal numeral system. Current Biology, in press.

FISCHER MH, CASTEL AD, DODD MD, and PRATT J. Perceiving numbers causes spatial shifts of attention. Nature Neuroscience, 6: 555-6, 2003.

GAINOTTI G, D'ERME P, and BARTOLOMEO P. Early orientation of attention toward the half space ipsilateral to the lesion in patients with unilateral brain damage. Journal of Neurology, Neurosurgery \& Psychiatry, 54: 1082-9, 1991.

HALSBAND U, GRUHN S, and ETTLINGER G. Unilateral spatial neglect and defective performance in one half of space. International Journal of Neuroscience, 28: 173-195, 1985.

HEINZE N and RIEDWYL H. How to win more : Strategies for increasing a lottery win. Natick, Mass: A K Peters, 1998.

HUBBARD EM, PIAZZA M, PINEL P, and DEHAENE S. Interactions between number and space in parietal cortex. Nature Reviews Neuroscience, 6: 435-48, 2005.

KUBOVY M. Response availability and the apparent spontaneity of numerical choices. Journal of Experimental Psychology: Human Perception and Performance, 3: 359-364, 1977.

LOETSCHER T, BOCKISCH C, and BRUGGER P. Looking for the answer: The mind's eye in number space. Neuroscience, 151: 725-729, 2008.

LOETSCHER T and BRUGGER P. Random number generation in neglect patients reveals enhanced response stereotypy, but no neglect in number space. Neuropsychologia, 47: 276-279, 2009.

MATTINGLEY JB, BRADSHAW JL, BRADSHAW JA, and NETTLETON NC. Residual rightward attentional bias after apparent recovery from right hemisphere damage: Implications for a multicomponent model of neglect. Journal of Neurology, Neurosurgery \& Psychiatry, 57: 597-604, 1994.

MEADOR KJ, LORING DW, BOWERS D, and HEILMAN KM. Remote memory and neglect syndrome. Neurology, 37: 522-6, 1987.

MOYER RS and LANDAUER TK. Time required for judgements of numerical inequality. Nature, 215: 151920, 1967.

NATALE E, POSTERARO L, PRIOR M, and MARZI CA. What kind of visual spatial attention is impaired in neglect? Neuropsychologia, 43: 1072-1085, 2005.

POSNER MI, WALKER JA, FRIEDRICH FJ, and RAFAL RD. Effects of parietal injury on covert orienting of attention. Journal of Neuroscience, 4: 1863-74, 1984.

RESTLE F. Speed of adding and comparing numbers. Journal of Experimental Psychology, 83: 274-278, 1970.

RODE G, ROSSETTI Y, PERENIN MT, and BOISSON D. Geographic information has to be spatialised to be neglected: A representational neglect case. Cortex, 40: 391-397, 2004.

SANDRINI M and RUSCONI E. A brain for numbers. Cortex, In Press.

SCOTT S, BARNARD P, and MAY J. Specifying executive representations and processes in number generation tasks. Quarterly Journal of Experimental Psychology: Section A, 54: 641-664, 2001. 
SIEROFF E, DECAIX C, CHOKRON S, and BARTOLOMEO P. Impaired orienting of attention in left unilateral neglect: A componential analysis. Neuropsychology, 21: 94-113, 2007.

UMILTÀ C, PRIFTIS K, and ZORZI M. The spatial representation of numbers: Evidence from neglect and pseudoneglect. Experimental Brain Research, 192: 561-569, 2009.

WOOD G and FISCHER MH. Numbers, space, and action - from finger counting to the mental number line and beyond. Cortex, 44: 353-358, 2008.

ZAMARIAN L, EGGER C, and DELAZER M. The mental representation of ordered sequences in visual neglect. Cortex, 43: 542-550, 2007.

ZORZI M, PRIFTIS K, MENEGHELLO F, MARENZI R, and UMILTA C. The spatial representation of numerical and non-numerical sequences: Evidence from neglect. Neuropsychologia, 44: 1061-1067, 2006.

ZORZI M, PRIFTIS K, and UMILTA C. Brain damage: Neglect disrupts the mental number line. Nature, 417 : $138-9,2002$ 


\section{Figure Legend:}

\section{Figure 1}

Frequencies of the generated 4-digit number lying between 1,000 and 10,000 (top panel), "imaginary lottery" indices (mid panel) and "physical lottery" indices (bottom panel) with the data of the neglect patients shown on the left, and the controls on the right side, respectively. The first two panels represent the selection bias in number space, the bottom panel the selection bias in physical space. For each of the three panels, the possible range of responses was split in 5 classes of equal length and the participants' answers were assigned accordingly. The most leftward bar in each graph thus denotes a strong bias towards the selection of smaller numbers in the numerical tasks and the left-side of physical space in the "physical lottery task" respectively. This bias gradually decreases from left to right, with the middle bar representing the frequency of responses around the center of the possible selection ranges and the most rightward bar denoting a strong bias towards large numbers and right-side of space. See main text for calculation of indices. 

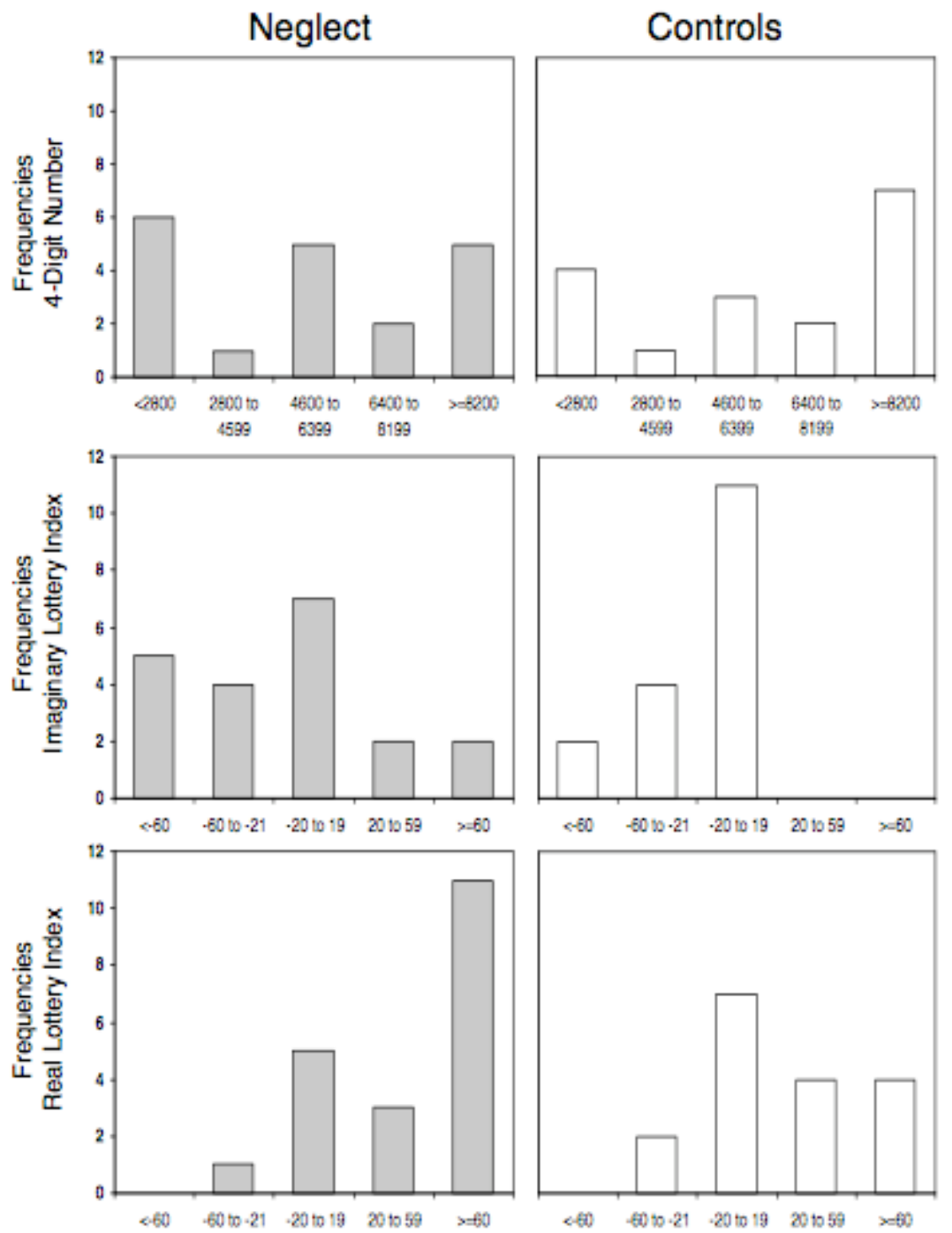